International Journal of Pure and Applied Mathematics

Volume 95 No. 1 2014, 1-11

ISSN: 1311-8080 (printed version); ISSN: 1314-3395 (on-line version)

url: http://www.ijpam.eu

doi: http://dx.doi.org/10.12732/ijpam.v95i1.1

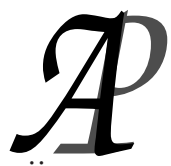

ijpam.eu

\title{
HYERS-ULAM-RASSIAS STABILITY OF FUNCTIONAL EQUATION IN NAB-SPACES
}

\author{
Dong Yun Shin ${ }^{1}$, Hassan Azadi Kenary ${ }^{2}$, N. Sahami ${ }^{3}$ \\ ${ }^{1}$ Department of Mathematics \\ College of Natural Science \\ University of Seoul \\ KOREA \\ ${ }^{2,3}$ Department of Mathematics \\ Beyza Branch \\ Islamic Azad University \\ Beyza, IRAN
}

\begin{abstract}
In this paper, using direct method we investigate the Hyers-UlamRassias stability of an additive functional equation in non-Archimedean Banach spaces (briefly, NAB-spaces).
\end{abstract}

AMS Subject Classification: 39B22, 39B52,39B22, 39B82, 46S10

Key Words: Hyers-Ulam-Rassias stability, non-Archimedean Banach spaces

\section{Introduction and Preliminaries}

A classical question in the theory of functional equations is the following: When is it true that a function which approximately satisfies a functional equation $D$ must be close to an exact solution of $D$ ?.

If the problem accepts a solution, we say that the equation $D$ is stable. The first stability problem concerning group homomorphisms was raised by Ulam [26] in 1940.

Received: April 29, 2013

(C) 2014 Academic Publications, Ltd.

$\S$ Correspondence author url: www.acadpubl.eu 
We are given a group $G$ and a metric group $G^{\prime}$ with metric $d(.,$.$) . Given \varepsilon>$ 0 , dose there exist a $\delta>0$ such that if $f: G \rightarrow G^{\prime}$ satisfies $d(f(x y), f(x) f(y))<$ $\delta$, for all $x, y \in G$, then a homomorphism $h: G \rightarrow G$; exists with $d(f(x), h(x))<$ $\varepsilon$ for all $x \in G$ ?

Ulam's problem was partially solved by Hyers [10] in 1941.

In 1978, Th. M. Rassias [18] formulated and proved the following theorem, which implies Hyers's Theorem as a special case. Suppose that $E$ and $F$ are real normed spaces with $F$ a complete normed space, $f: E \rightarrow F$ is a mapping such that for each fixed $x \in E$ the mapping $t \rightarrow f(t x)$ is continuous on $R$, and let there exist $\varepsilon \geq 0$ and $p \in[0,1)$ such that for all $x, y \in E$

$$
\|f(x+y)-f(x)-f(y)\| \leq \varepsilon\left(\|x\|^{p}+\|y\|^{p}\right)
$$

Then there exists a unique linear mapping $T: E \rightarrow F$ such that such that for all $x \in E$

$$
\|f(x)-T(x)\| \leq \frac{\varepsilon\|x\|^{p}}{1-2^{p-1}}
$$

The case of the existence of a unique additive mapping had been obtained by T. Aoki [2], as it is recently noticed by Lech Maligranda. However, Aoki [2] had claimed the existence of a unique linear mapping, that is not true because he did not allow the mapping $f$ to satisfy some continuity assumption. Th.M. Rassias [18], who independently introduced the unbounded Cauchy difference was the first to prove that there exists a unique linear mapping $\mathrm{T}$ satisfying

$$
\|f(x)-T(x)\| \leq \frac{\epsilon\|x\|^{p}}{1-2^{p-1}} \quad x \in E
$$

In 1990, Th.M. Rassias [19] during the 27th International Symposium on Functional Equations asked the question whether such a theorem can also be proved for $p \geq 1$. In 1991, Z. Gajda [8] following the same approach as in Th. M. Rassias [24], gave an affirmative solution to this question for $p>1$. It was proved by Z. Gajda [8], as well as by Th. M. Rassias and P. Šemrl [20] that one can not prove a Th. M. Rassias type theorem when $p=1$. In 1994, P. Găvruta [9] provided a further generalization of Th. M. Rassias theorem in which he replaced the bound $\varepsilon\left(\|x\|^{p}+\|\| y \|^{p}\right)$ by a general control function $\psi(x, y)$ for the existence of a unique linear mapping.

The functional equation

$$
f(x+y)+f(x-y)=2 f(x)+2 f(y)
$$

is called the quadratic functional equation. In particular, every solution of the quadratic functional equation is said to be a quadratic mapping. A generalized 
Hyers-Ulam stability problem for the quadratic functional equation was proved by Skof [25] for mappings $f: X \rightarrow Y$, where $X$ is a normed space and $Y$ is a Banach space. Cholewa [6] noticed that the theorem of Skof is still true if the relevant domain $\mathrm{X}$ is replaced by an Abelian group. In [7], Czerwik proved the generalized Hyers-Ulam stability of the quadratic functional equation.

During the last decades several stability problems of functional equations have been investigated by a number of mathematicians( [1]-[5], [11]-[24]).

Definition 1.1. By a non-Archimedean field we mean a field $\mathbb{K}$ equipped with a function(valuation) $||:. \mathbb{K} \rightarrow[0, \infty)$ such that for all $r, s \in \mathbb{K}$, the following conditions hold:

(i) $|r|=0$ if and only if $r=0$

(ii) $|r s|=|r||s|$

(iii) $|r+s| \leq \max \{|r|,|s|\}$.

Definition 1.2. Let $X$ be a vector space over a scalar field $\mathbb{K}$ with a non-Archimedean non-trivial valuation |.| . A function $\|\|:. X \rightarrow \mathbb{R}$ is a nonArchimedean norm (valuation) if it satisfies the following conditions:

(i) $\|x\|=0$ if and only if $x=0$

(ii) $\quad\|r x\|=|r|\|x\| \quad(r \in \mathbb{K}, x \in X)$

(iii) The strong triangle inequality( ultrametric); namely

$$
\|x+y\| \leq \max \{\|x\|,\|y\|\} . \quad x, y \in X
$$

Then $(X,\|\|$.$) is called a non-Archimedean space.$

Due to the fact that

$$
\left\|x_{n}-x_{m}\right\| \leq \max \left\{\left\|x_{j+1}-x_{j}\right\|: m \leq j \leq n-1\right\} \quad(n>m)
$$

Definition 1.3. A sequence $\left\{x_{n}\right\}$ is Cauchy if and only if $\left\{x_{n+1}-x_{n}\right\}$ converges to zero in a non-Archimedean space. By a complete non-Archimedean space we mean one in which every Cauchy sequence is convergent.

Example 1.1. Fix a prime number $p$. For any nonzero rational number $x$, there exists a unique integer $n_{x} \in \mathbb{Z}$ such that $x=\frac{a}{b} p^{n_{\times}}$, where a and $\mathrm{b}$ are integers not divisible by $p$. Then $|x|_{p}:=p^{-n_{\times}}$defines a non-Archimedean norm on $\mathbb{Q}$. The completion of $\mathbb{Q}$ with respect to the metric $d(x, y)=|x-y|_{p}$ is denoted by $\mathbb{Q}_{p}$ which is called the $p$-adic number field. In fact, $\mathbb{Q}_{p}$ is the set of all formal series $x=\sum_{k \geq n_{\mathrm{x}}}^{\infty} a_{k} p^{k}$ where $\left|a_{k}\right| \leq p-1$ are integers. The addition 
and multiplication between any two elements of $\mathbb{Q}_{p}$ are defined naturally. The norm $\left|\sum_{k \geq n_{\mathrm{x}}}^{\infty} a_{k} p^{k}\right|_{p}=p^{-n_{\mathrm{x}}}$ is a non-Archimedean norm on $\mathbb{Q}_{p}$ and it makes $\mathbb{Q}_{p}$ a locally compact filed.

Definition 1.4. Let $X$ be a set. A function $d: X \times X \rightarrow[0, \infty]$ is called a generalized metric on $X$ if $d$ satisfies the following conditions:

(a) $d(x, y)=0$ if and only if $x=y$ for all $x, y \in X$;

(b) $d(x, y)=d(y, x)$ for all $x, y \in X$;

(c) $d(x, z) \leq d(x, y)+d(y, z)$ for all $x, y, z \in X$.

Theorem 1.1. Let $(X, d)$ be a complete generalized metric space and $J: X \rightarrow X$ be a strictly contractive mapping with Lipschitz constant $L<1$. Then, for all $x \in X$, either

$$
d\left(J^{n} x, J^{n+1} x\right)=\infty
$$

for all nonnegative integers $n$ or there exists a positive integer $n_{0}$ such that:

(a) $d\left(J^{n} x, J^{n+1} x\right)<\infty$ for all $n_{0} \geq n_{0}$;

(b) the sequence $\left\{J^{n} x\right\}$ converges to a fixed point $y^{*}$ of $J$;

(c) $y^{*}$ is the unique fixed point of $J$ in the set $Y=\left\{y \in X: d\left(J^{n_{0}} x, y\right)<\right.$ $\infty\}$;

(d) $d\left(y, y^{*}\right) \leq \frac{1}{1-L} d(y, J y)$ for all $y \in Y$.

In this paper, we prove the generalized Hyers-Ulam stability of the following functional equation

$$
f(f(x)-f(y))=f(x+y)+f(x-y)-f(x)-f(y)
$$

in non-Archimedean normed spaces. In the rest of the paper let $|2| \neq 1$.

\section{Non-Archimedean Stability of Eq. (1.3): A Direct Method}

Throughout this section, using direct method we prove the generalized HyersUlam stability of composite functional equation (1.3) in non-Archimedean spaces.

Theorem 2.1. Let $G$ is an additive semigroup and $X$ is a complete nonArchimedean space. Assume that $\varphi: G^{2} \rightarrow[0,+\infty)$ be a function such that

$$
\lim _{n \rightarrow \infty} \frac{\varphi\left(2^{n} x, 2^{n} y\right)}{|2|^{n}}=0
$$


for all $x, y \in G$. Let for all $x \in G$

$$
\Phi(x)=\operatorname{Sup}_{k \geq 0}\left\{\frac{\varphi\left(2^{k} x, 2^{k} x\right)}{|2|^{k}} ; k \in \mathbb{N} \cup\{0\}\right\}
$$

exists. Suppose that $f: G \rightarrow X$ be a mapping satisfying the inequality

$$
\|f(f(x)-f(y))-f(x+y)-f(x-y)+f(x)+f(y)\| \leq \varphi(x, y)
$$

for all $x, y \in G$. Then the limit

$$
A(x):=\lim _{n \rightarrow \infty} \frac{f\left(2^{n} x\right)}{2^{n}}
$$

exist for all $x \in G$ and $A: G \rightarrow X$ is an additive mapping satisfying

$$
\|f(x)-A(x)\| \leq \frac{1}{|2|} \Phi(x)
$$

for all $x \in G$. Moreover, if

$$
\lim _{j \rightarrow \infty} \lim _{n \rightarrow \infty} \max \left\{\frac{\varphi\left(2^{k} x, 2^{k} x\right)}{|2|^{k}} ; j \leq k<n+j\right\}=0
$$

Then $A$ is the unique mapping satisfying (2.4).

Proof. Putting $y=x$ in (2.3), we have

$$
\left\|\frac{f(2 x)}{2}-f(x)\right\|_{X} \leq \frac{1}{|2|} \varphi(x, x)
$$

Replacing $x$ by $2^{n} x$ in (2.5), we get

$$
\left\|\frac{f\left(2^{n+1} x\right)}{2^{n+1}}-\frac{f\left(2^{n} x\right)}{2^{n}}\right\|_{X} \leq \frac{\varphi\left(2^{n} x, 2^{n} x\right)}{|2|^{n+1}}
$$

It follows from (2.1) and (2.6) that the sequence $\left\{\frac{f\left(2^{n} x\right)}{2^{n}}\right\}_{n=1}^{\infty}$ is a Cauchy sequence. Since $X$ is complete, so $\left\{\frac{f\left(2^{\mathrm{n}} x\right)}{2^{\mathrm{n}}}\right\}_{n=1}^{\infty}$ is convergent. Set

$$
A(x):=\lim _{n \rightarrow \infty} \frac{f\left(2^{n} x\right)}{2^{n}} .
$$

Using induction we see that

$$
\left\|\frac{f\left(2^{n} x\right)}{2^{n}}-f(x)\right\|_{X} \leq \frac{1}{|2|} \max \left\{\frac{\varphi\left(2^{k} x, 2^{k} x\right)}{|2|^{k}} ; 0 \leq k<n\right\} .
$$


Indeed, (2.7) holds for $n=1$ by (2.5). Let, (2.7) holds for $n$, so by (2.6), we obtain

$$
\begin{aligned}
& \left\|\frac{f\left(2^{n+1} x\right)}{2^{n+1}}-f(x)\right\|_{X}=\left\|\frac{f\left(2^{n+1} x\right)}{2^{n+1}} \pm \frac{f\left(2^{n} x\right)}{2^{n}}-f(x)\right\|_{X} \\
\leq & \max \left\{\left\|\frac{f\left(2^{n+1} x\right)}{2^{n+1}}-\frac{f\left(2^{n} x\right)}{2^{n}}\right\|_{X},\left\|\frac{f\left(2^{n} x\right)}{2^{n}}-f(x)\right\|_{X}\right\} \\
\leq & \frac{1}{|2|} \max \left\{\frac{\varphi\left(2^{n} x, 2^{n} x\right)}{|2|^{n}}, \max \left\{\frac{\varphi\left(2^{k} x, 2^{k} x\right)}{|2|^{k}} ; 0 \leq k<n\right\}\right\} \\
= & \frac{1}{|2|} \max \left\{\frac{\varphi\left(2^{k} x, 2^{k} x\right)}{\mid 2^{k}} ; 0 \leq k<n+1\right\} .
\end{aligned}
$$

So for all $n \in \mathbb{N}$ and all $x \in G,(2.7)$ holds. By taking $n$ to approach infinity in (2.8), one obtains (2.4).

If $L$ is another mapping satisfies (2.4), then for $x \in G$, we get

$$
\begin{aligned}
\|A(x)-L(x)\|_{X} & =\lim _{k \rightarrow \infty}\left\|\frac{A\left(2^{k} x\right)}{2^{k}}-\frac{L\left(2^{k} x\right)}{2^{k}}\right\|_{X} \\
& =\lim _{k \rightarrow \infty}\left\|\frac{A\left(2^{k} x\right)}{2^{k}} \pm \frac{f\left(2^{k} x\right)}{2^{k}}-\frac{L\left(2^{k} x\right)}{2^{k}}\right\|_{X} \\
& \leq \lim _{k \rightarrow \infty} \max \left\{\left\|\frac{A\left(2^{k} x\right)-f\left(2^{k} x\right)}{2^{k}}\right\|_{X},\left\|\frac{f\left(2^{k} x\right)-L\left(2^{k} x\right)}{2^{k}}\right\|_{X}\right\} \\
& \leq \lim _{j \rightarrow \infty} \lim _{n \rightarrow \infty} \max \left\{\frac{\varphi\left(2^{k} x, 2^{k} x\right)}{|2|^{k}} ; j \leq k<n+j\right\} \\
& =0
\end{aligned}
$$

Therefore $A=L$. This completes the proof.

Corollary 2.1. Let $\xi:[0, \infty) \rightarrow[0, \infty)$ be a function satisfying

$$
\xi(|2| t) \leq \xi(|2|) \lambda(t), \quad \xi(|2|)<|2|
$$

for all $t \geq 0$. Let $\delta>0$ and $f: G \rightarrow X$ is a mapping satisfying the inequality

$$
\|f(f(x)-f(y))-f(x+y)-f(x-y)+f(x)+f(y)\| \leq \delta(\xi(\|x\|)+\xi(\|y\|))
$$

for all $x, y \in G$. Then the limit $A(x)=\lim _{n \rightarrow \infty} \frac{f\left(2^{\mathrm{n}} x\right)}{2^{\mathrm{n}}}$ exists for all $x \in G$ and $A: G \rightarrow X$ is a unique additive mapping such that

$$
\|f(x)-A(x)\| \leq \frac{2 \delta \xi(\|x\|)}{|2|}
$$

for all $x \in G$. 
Proof. Defining $\varphi: G^{2} \rightarrow[0, \infty)$ by $\varphi(x, y):=\delta(\xi(\|x\|)+\xi(\|y\|))$. Since $\frac{\xi(|2|)}{|2|}<1$, we have

$$
\lim _{n \rightarrow \infty} \frac{\varphi\left(2^{n} x, 2^{n} y\right)}{|2|^{n}} \leq \lim _{n \rightarrow \infty}\left(\frac{\xi(|2|)}{|2|}\right)^{n} \varphi(x, y)=0
$$

for all $x, y \in G$. Also for all $x \in G$

$$
\Phi(x)=\operatorname{Sup}_{k \geq 0}\left\{\frac{\varphi\left(2^{k} x, 2^{k} x\right)}{|2|^{k}} ; k \in \mathbb{N} \cup\{0\}\right\}=\varphi(x, x)=2 \delta \xi(\|x\|)
$$

exists for all $x \in G$. On the other hand

$$
\lim _{j \rightarrow \infty} \lim _{n \rightarrow \infty} \max \left\{\frac{\varphi\left(2^{k} x, 2^{k} x\right)}{|2|^{k}} ; j \leq k<n+j\right\}=\lim _{j \rightarrow \infty} \frac{\varphi\left(2^{j} x, 2^{j} x\right)}{|2|^{j}}=0 .
$$

Applying Theorem 2.1, then we get the desired result.

Theorem 2.2. Let $G$ is an additive semigroup and $X$ is a complete nonArchimedean space. Assume that $\varphi: G^{2} \rightarrow[0,+\infty)$ be a function such that

$$
\lim _{n \rightarrow \infty}|2|^{n} \varphi\left(\frac{x}{2^{n}}, \frac{y}{2^{n}}\right)=0
$$

for all $x, y \in G$. Let for each $x \in G$

$$
\Phi(x)=\operatorname{Sup}_{k \geq 0}\left\{|2|^{k} \varphi\left(\frac{x}{2^{k+1}}, \frac{x}{2^{k+1}}\right) ; k \in \mathbb{N} \cup\{0\}\right\}
$$

exists. Suppose that $f: G \rightarrow X$ is a mapping satisfying the inequality (2.3). Then the limit

$$
A(x):=\lim _{n \rightarrow \infty} 2^{n} f\left(\frac{x}{2^{n}}\right)
$$

exist for all $x \in G$ and $A: G \rightarrow X$ is an additive mapping satisfying

$$
\|f(x)-A(x)\| \leq \Phi(x)
$$

for all $x \in G$. Moreover, if

$$
\lim _{j \rightarrow \infty} \lim _{n \rightarrow \infty} \max \left\{|2|^{k} \varphi\left(\frac{x}{2^{k+1}}, \frac{x}{2^{k+1}}\right) ; j \leq k<n+j\right\}=0
$$

Then $A$ is the unique mapping satisfying (2.12). 
Proof. By (??), we have

$$
\left\|f(x)-2 f\left(\frac{x}{2}\right)\right\|_{X} \leq \zeta\left(\frac{x}{2}, \frac{x}{2}\right)
$$

for all $x \in G$. Replacing $x$ by $\frac{x}{2^{n}}$ in $(2.14)$, we get

$$
\left\|2^{n} f\left(\frac{x}{2^{n}}\right)-2^{n+1} f\left(\frac{x}{2^{n+1}}\right)\right\|_{X} \leq|2|^{n} \varphi\left(\frac{x}{2^{n+1}}, \frac{x}{2^{n+1}}\right)
$$

It follows from (2.10) and (2.15) that the sequence $\left\{2^{n} f\left(\frac{x}{2^{n}}\right)\right\}_{n=1}^{\infty}$ is a Cauchy sequence. Since $X$ is complete, so $\left\{2^{n} f\left(\frac{x}{2^{n}}\right)\right\}_{n=1}^{\infty}$ is convergent. It follows from (2.15) that

$$
\begin{aligned}
& \left\|2^{n} f\left(\frac{x}{2^{n}}\right)-2^{p} f\left(\frac{x}{2^{p}}\right)\right\|_{X}=\left\|\sum_{k=p}^{n-1} 2^{k+1} f\left(\frac{x}{2^{k+1}}\right)-2^{k} f\left(\frac{x}{2^{k}}\right)\right\|_{X} \\
\leq & \max \left\{\left\|2^{k+1} f\left(\frac{x}{2^{k+1}}\right)-2^{k} f\left(\frac{x}{2^{k}}\right)\right\|_{X} ; p \leq k<n-1\right\} \\
\leq & \max \left\{|2|^{k+1} \varphi\left(\frac{x}{2^{k+1}}, \frac{x}{2^{k+1}}\right) ; p \leq k<n-1\right\}
\end{aligned}
$$

for all $x \in G$ all non-negative integer $n, p$ with $n>p \geq 0$. Letting $p=0$ and passing the limit $n \rightarrow \infty$ in the last inequality, we obtain (2.12). The rest of the proof is similar to the proof of Theorem 2.1.

Corollary 2.2. Let $\xi:[0, \infty) \rightarrow[0, \infty)$ be a function satisfying

$$
\xi\left(|2|^{-1} t\right) \leq \xi\left(|2|^{-1}\right) \lambda(t), \quad \xi\left(|2|^{-1}\right)<|2|^{-1}
$$

for all $t \geq 0$. Let $\delta>0$ and $f: G \rightarrow X$ is a mapping satisfying the inequality (2.9). Then the limit $A(x)=\lim _{n \rightarrow \infty} 2^{n} f\left(\frac{x}{2^{n}}\right)$ exists for all $x \in G$ and $A: G \rightarrow$ $X$ is a unique additive mapping such that

$$
\|f(x)-A(x)\| \leq \frac{2 \xi(\|x\|)}{|2|}
$$

for all $x \in G$.

Proof. Defining $\varphi: G^{2} \rightarrow[0, \infty)$ by $\varphi(x, y):=\delta(\xi(\|x\|)+\xi(\|y\|))$. Proceeding as in the proof of the Corollary 2.1, we have $\lim _{n \rightarrow \infty} \frac{\varphi\left(2^{\mathrm{n}} x, 2^{\mathrm{n}} y\right)}{|2|^{\mathrm{n}}}=0$ for all $x, y \in G$. Also for all $x \in G$

$$
\Phi(x)=\operatorname{Sup}_{k \geq 0}\left\{|2|^{k} \varphi\left(\frac{x}{2^{k+1}}, \frac{x}{2^{k+1}}\right) ; k \in \mathbb{N} \cup\{0\}\right\}=\varphi\left(\frac{x}{2}, \frac{x}{2}\right)=\frac{2 \xi(\|x\|)}{|2|}
$$

exists for all $x \in G$. Applying Theorem 2.2, then we get the desired result. 


\section{Acknowledgments}

D.Y. Shin was supported by Basic Science Research Program through the National Research Foundation of Korea funded by the Ministry of Education, Science and Technology (NRF-2010-0021792).

\section{References}

[1] L. M. Arriola and W. A. Beyer, Stability of the Cauchy functional equation over p-adic fields, Real Anal. Exchange 31 (2005/06), no. 1, 125-132.

[2] T. Aoki, On the stability of the linear transformationin Banach spaces, J. Math. Soc. Japan, 2 (1950), 64-66.

[3] H. Azadi Kenary, Hyres-Rassias Stability of The Pexiderial Functional Equation, Italian Journal of Pure and Applied Mathematics (in press).

[4] H. Azadi Kenary, The Probabilistic Stability of a Pexiderial Functional Equation in Random Normed Spaces, Rendiconti Del Circolo Mathematico Di Palermo (in press).

[5] Hassan Azadi Kenary and Yeol Je Cho, Stability of mixed additivequadratic Jensen type functional equation in various spaces, Computer and Mathematics with Applications, (2011), doi:10.1016/j.camwa.2011.03.024.

[6] P. W. Cholewa, Remarks on the stability of functional equations, Aequationes Mathematicae, 27(1984)76-86.

[7] S. Czerwik, Functional Equations and Inequalities in Several Variables, World Scientific, River Edge, NJ, 2002.

[8] Z. Gajda, On stability of additive mappings, Internat. J. Math. Math. Sci., 14 (1991), 431-434.

[9] P. Găvruta, A generalization of the Hyers-Ulam-Rassias stability of approximately additive mappings, J. Math. Anal. Appl. 184 (1994), no. 3, 431-436.

[10] D. H. Hyers, On the stability of the linear functional equation, Proc. Nat. Acad. Sci. U.S.A. 27 (1941), 222-224.

[11] D. H. Hyers, G. Isac, and Th. M. Rassias, Stability of Functional Equations in Several Variables, Birkhauser, Basel, 1998. 
[12] D. Mihet and V. Radu, On the stability of the additive Cauchy functional equation in random normed spaces, Journal of Math. Anal. and Appl., $343(2008) 567-572$.

[13] Alireza Kamel Mirmostafaee , Approximately Additive Mappings in NonArchimedean Normed Spaces, Bull. Korean Math. Soc. 46(2009), No.2, pp. 387-400.

[14] C. Park, Fuzzy stability of a functional equation associated with inner product spaces, Fuzzy Sets and Systems 160(2009), 1632-1642.

[15] C. Park, Generalized Hyers-Ulam-Rassias stability of $n$-sesquilinearquadratic mappings on Banach modules over $C^{*}$-algebras, J. Comput. Appl. Math. 180 (2005), 279-291.

[16] C. Park, J. Hou, and S. Oh, Homomorphisms between JC-algebras and Lie $C^{*}$-algebras, Acta Math. Sin. (Engl. Ser.) 21 (2005), no. 6, 1391-1398.

[17] C. Park, On the stability of the quadratic mapping in Banach modules, J. Math. Anal. Appl., 276 (2002), 135-144.

[18] Th. M. Rassias, On the stability of the linear mapping in Banach spaces, Proc. Amer. Math. Soc. 72 (1978), no. 2, 297-300.

[19] Th. M. Rassias, Problem 16; 2, Report of the 27th International Symposium Functional Equations, Aequationes Math., 39 (1990), 292-309.

[20] Th. M. Rassias, P. Šemrl, On the behaviour of mappings which do not satisfy Hyers-Ulam stability, Proc. Amer. Math. Soc., 114 (1992), 989-993.

[21] R. Saadati, M. Vaezpour and Y.J. Cho, A note to paper "On the stability of cubic mappings and quartic mappings in random normed spaces", J. Ineq. Appl., Volume 2009, Article ID 214530, doi: 10.1155/2009/214530.

[22] R. Saadati and C. Park, Non-Archimedean $\mathcal{L}$-fuzzy normed spaces and stability of functional equations (in press).

[23] R. Saadati, M. Vaezpour and Y. J. Cho, A note to paper "On the stability of cubic mappings and quartic mappings in random normed spaces", J. Ineq. Appl., Volume 2009, Article ID 214530, doi: 10.1155/2009/214530.

[24] Reza Saadati, M. M. Zohdi, and S. M. Vaezpour, Nonlinear L-Random Stability of an ACQ Functional Equation, Journal of Inequalities and Applications, Volume 2011, Article ID 194394, 23 pages, doi:10.1155/2011/194394. 
[25] F. Skof, Local properties and approximation of operators, Rend. Sem. Mat. Fis. Milano 53 (1983), 113-129.

[26] S. M. Ulam, Problems in Modern Mathematics, Science Editions, John Wiley and Sons, 1964. 
